\title{
Value of Measuring Myocardial Performance Index by Tissue Doppler Echocardiography in Normal and Diseased Heart
}

\author{
Tarkan TeKTen, ${ }^{1} \mathrm{MD}$, Alper O. OnBasili, ${ }^{1} \mathrm{MD}$, Ceyhun Ceyhan, ${ }^{1} \mathrm{MD}$, \\ Selim ÜNAL, ${ }^{1} \mathrm{MD}$, and Berent DisCIGIL, ${ }^{2} \mathrm{MD}$
}

\begin{abstract}
SUMMARY
Myocardial performance index (MPI) is a simple and reproducible method for the assessment of overall cardiac function. In this study, we evaluated the applicability and usefulness of pulsed-wave tissue Doppler echocardiography (TDE) as a tool to calculate MPI in comparision with a conventional Doppler method.

Twenty-five patients with previous myocardial infarction (MI) and 15 healthy subjects were included. In order to calculate MPI by TDE, isovolumetric contraction time (IVCT), isovolumetric relaxation time (IVRT), and ejection time (ET) were measured at four different sites in the mitral annulus: septum, lateral, anterior, and inferior. MPI was calculated by dividing the sum of IVCT and IVRT by ET at each site of measurement. The mean MPI value was found by dividing the sum of these MPI values into four. The same parameters were measured using the mitral inflow and left ventricular outflow velocity time intervals by a conventional method.

MPI by TDE correlated well with conventional MPI in healthy subjects $(r=0.81, P$ $<0.0001)$. Although it continued to be statistically significant, the correlation between the two methods of measuring MPI, TDE and conventional, in patients with previous MI was not as high as it was in healthy individuals $(P<0.001, r=0.62)$. This is due to the lesser degree of correlation between IVRT and IVCT values obtained by TDE and the conventional method ( $r=0.49, P<0.05 ; r=0.56, P<0.05$; respectively).

In conclusion, this study has demonstrated that MPI could be measured by tissue Doppler and that it correlated well with conventional MPI. Moreover, MPI by TDE has the advantage of assessing both regional and global myocardial performance. (Jpn Heart J 2003; 44: 403-416)
\end{abstract}

Key words: Myocardial performance index, Tissue Doppler echocardiography

THE conventional echocardiographic methods for assessing cardiac function are unable to distinguish, in one single scan, the individual phases of the cardiac

From the ${ }^{1}$ Department of Cardiology, ${ }^{2}$ Department of Cardiovascular Surgery, Adnan Menderes University School of Medicine, Aydin, Turkey.

Address for correspondence: Tarkan Tekten, MD, Department of Cardiology, Adnan Menderes University School of Medicine, Aydin 09100, Turkey.

This study was presented in part in the $14^{\text {th }}$ World Congress of Cardiology.

Received for publication June 5, 2002.

Revised and accepted October 17, 2002. 
cycle. ${ }^{1)}$ Moreover, regional myocardial abnormalities in these phases cannot be identified. Tissue Doppler echocardiography (TDE) is a new technique that records myocardial velocities during the cardiac cycle. ${ }^{2,3)}$ Quantitative recording of systolic and diastolic velocities can be done by TDE, which can precisely differentiate phases of the cardiac cycle.

The recently proposed Doppler derived myocardial performance index (MPI), which combines systolic and diastolic time intervals, has been reported to be a simple and reproducible index for assessing left ventricular (LV) function that has been shown to be independent of heart rate and blood pressure. ${ }^{4-6)} \mathrm{We}$ hypothesize that MPI could be determined by pulsed-wave TDE and may be more sensitive than conventional MPI in predicting LV function, especially in the presence of regional wall motion abnormality, as is the case in patients with previous myocardial infarction (MI). The purpose of this study was to evaluate the applicability and usefulness of pulsed-wave TDE as a tool to calculate MPI in comparision with a conventional Doppler method.

\section{MeThods}

Study population: Twenty-five patients with previous MI (22 males and 3 females) evidenced by electrocardiographic findings and the presence of wall motion abnormality on a two-dimensional (2D) echocardiographic study were studied. Fifteen healthy subjects (11 males, 4 females) without a history of cardiac disease or systemic hypertension and having normal findings on physical examination, chest roentgenography, electrocardiography, and 2-D and Doppler echocardiography served as controls. All study patients were in sinus rhythm. Of the 25 patients with previous MI, 14 had anterior MI, 11 had inferior MI, 9 had systemic hypertension, and 5 had diabetes mellitus. Mitral regurgitation was present in 12 of these patients, and all were graded as mild. Exclusion criteria included inadequate visualization, presence of dilated cardiomyopathy, LV hypertrophy, pericardial disease, infiltrative myocardial disease, and the presence of left bundle branch block on the electrocardiogram.

Echocardiography: The patients were examined in the left lateral decubitis position with a Hewlett-Packard Sonos 5500 (Andover, Massachusetts) phased-array system equipped with tissue Doppler technology. Measurements were made according to the recommendations of the American Society of Echocardiography. ${ }^{7)}$ The ejection fraction was calculated from apical 4- and 2-chamber views with Simpson's method. The mitral inflow velocity pattern was recorded with the pulsed-wave Doppler sample volume positioned between the tips of the mitral leaflets. The LV outflow pattern was recorded from the apical 5-chamber view with the pulsed wave Doppler sample volume positioned just below the aor- 
tic valve. Three consecutive beats were measured and averaged for each parameter. Two-dimensional and Doppler tracings were recorded over five cardiac cycles at a sweep speed of $100 \mathrm{~mm} / \mathrm{s}$.

Doppler measurements: Mitral inflow and left ventricular outflow velocity-time intervals were used to measure Doppler time intervals: isovolumetric contraction (IVCT), relaxation time (IVRT), and ejection time (ET) as demonstrated in Figure 1. The interval 'a' from the cessation to the onset of mitral inflow was equal to the sum of IVCT, ET, and IVRT. Left ventricular ET ' $\mathrm{b}$ ' was the duration of the left ventricular velocity profile. Thus, the sum of IVCT and IVRT was obtained by subtracting ' $b$ ' from ' $a$ '. The MPI was calculated as ( $a-b) / b$. IVRT was calculated by subtracting the interval ' $\mathrm{d}$ ', between the $\mathrm{R}$ wave and the cessation of left ventricular outflow, from the interval ' $c$ ', between the $\mathrm{R}$ wave and the onset of mitral inflow. ${ }^{9)}$ IVCT was calculated by subtracting IVRT from ' $a$ '- 'b'.

Tissue doppler echocardiography: The pulsed-wave TDE was performed by activating the tissue Doppler function in the same echocardiographic machine. Images were acquired using a variable frequency phased-array transducer (2.0 to 4.0 MHz). The filter settings were kept low, and gains were adjusted to the mini-

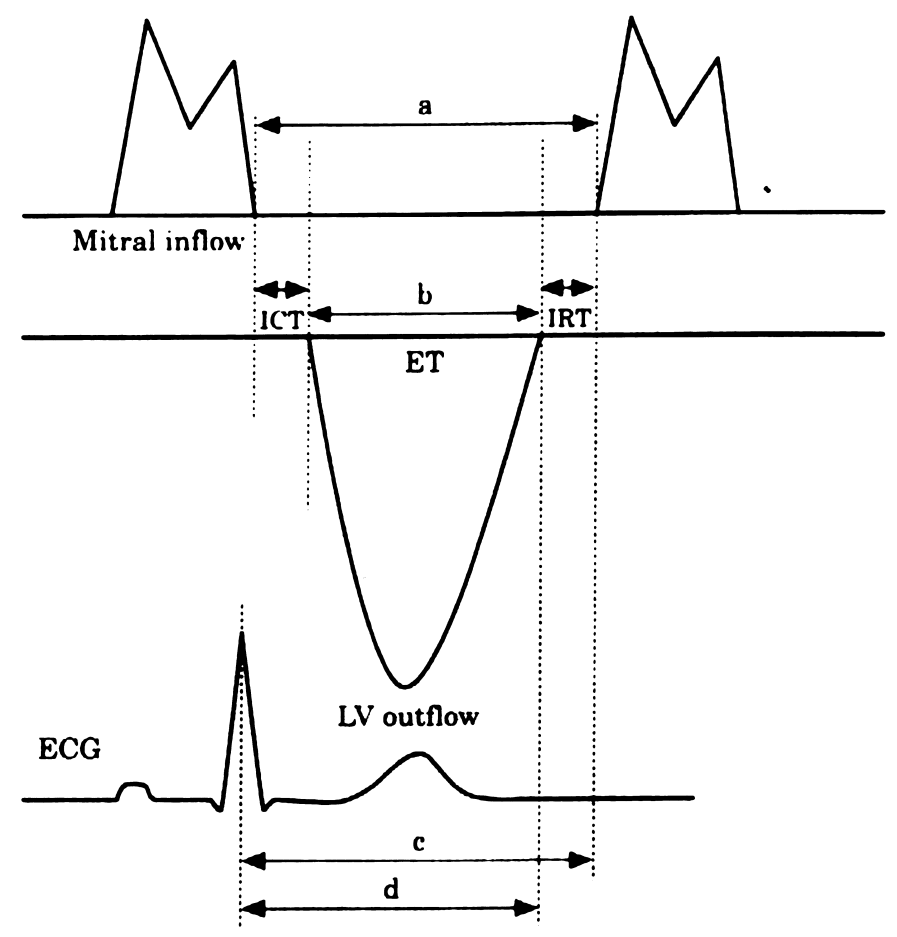

Figure 1. Doppler time intervals were measured from mitral inflow and left ventricular outflow velocity-time intervals. 
mal optimal level to minimize noise and eliminate the signals produced by the transmitral flow. A $1.7 \mathrm{~mm}$ sample volume was used.

Sample volumes were placed at four different sites in the mitral annulus, corresponding to the septum, lateral, anterior, and inferior sites, in order to record major velocity time intervals: IVCT, IVRT, and ET (Figure 2). The pulsed-wave TDE tracings were recorded over five cardiac cycles at a sweep speed of $100 \mathrm{~mm} /$ $s$ and three of them were used for calculation.

TDE velocity time intervals were measured from the sites at the mitral annulus as demonstrated in Figure 3. The interval (a'), from the R wave to the onset of diastolic velocity, was equal to the sum of IVCT, ET, and IVRT. Left ventricular ET (b') was the duration of the systolic velocity profile. Thus, the sum of IVCT and IVRT was obtained by subtracting (b') from (a'). The MPI was calculated as (a'-b')/b'. IVRT was calculated by subtracting the interval (d'), between the $R$ wave and the cessation of systolic velocity, from the interval (c'), between the $R$ wave and the onset of diastolic velocity. IVCT was calculated by subtracting IVRT from (a'- b').

Reproducibility: Intra-observer variability was assessed in 10 patients by repeating the measurements on two occasions (1-12 days apart) under the same basal conditions. To test the interobserver variability, the measurements were performed off-line from video recordings by a second observer who was unaware of the results of the first examination. Variability was calculated as the mean per-

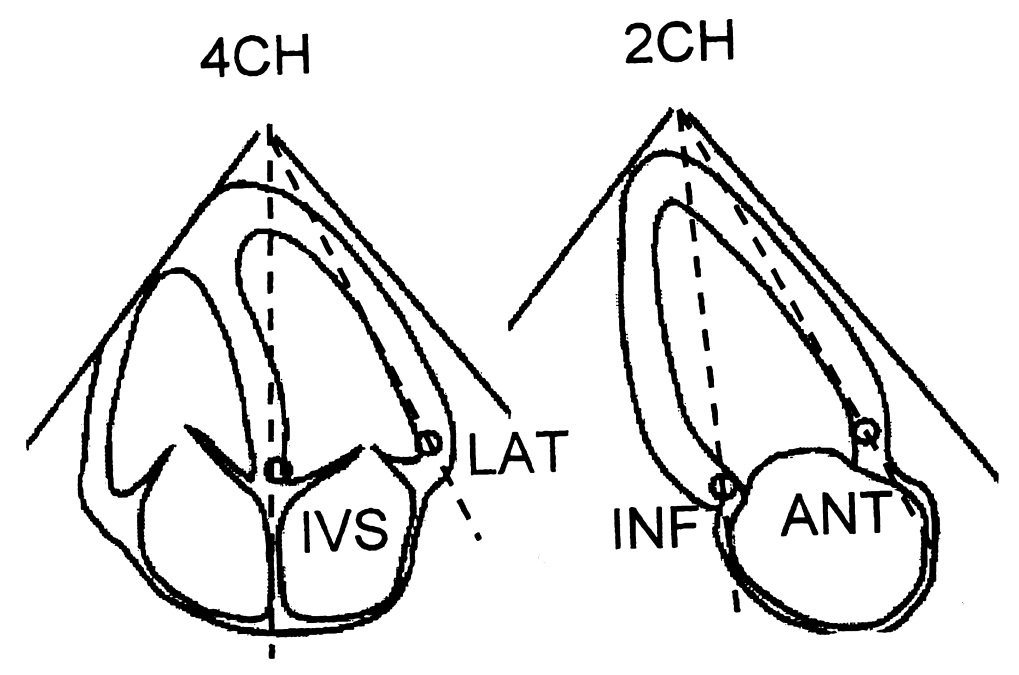

Figure 2. Position of the sample volumes in each cross-sectional view. ANT $=$ anterior wall $; 2 \mathrm{CH}=$ apical 2-chamber view; $4 \mathrm{CH}$ = apical 4-chamber view; INF = inferior wall; IVS = interventricular septum; LAT $=$ lateral wall. 


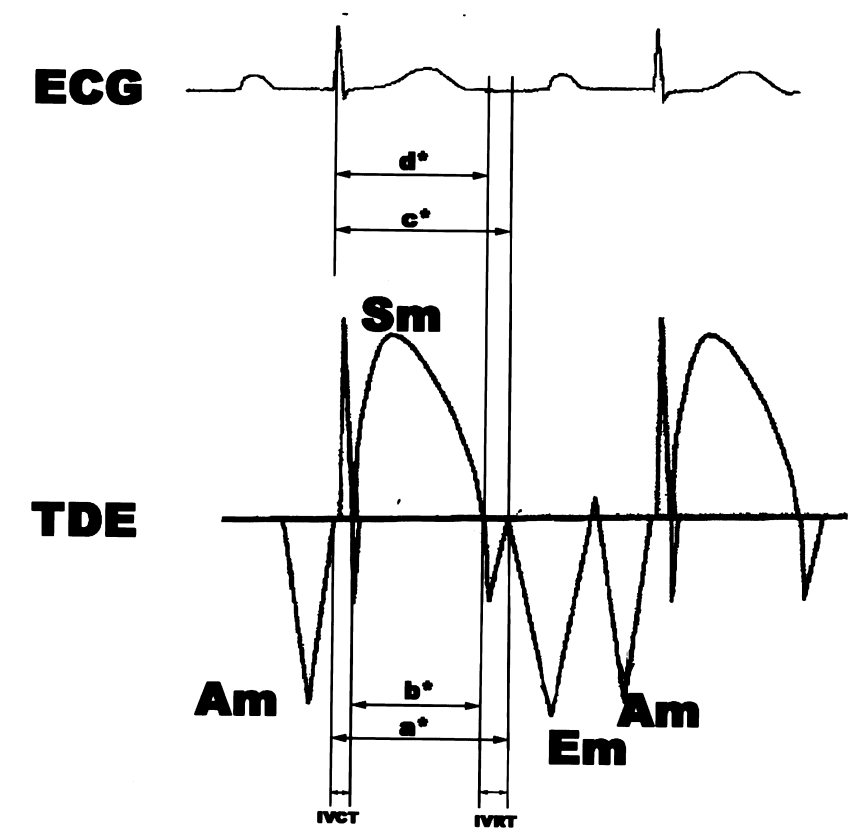

Figure 3. Tissue Doppler echocardiography time intervals were measured from the mitral annular tissue Doppler velocity time intervals.

centage error, derived as the difference between the two sets of measurements, divided by the mean observations.

Statistics: Data are expressed as the mean \pm SD. Groups were compared by the Kruskal-Wallis and Mann-Whitney U tests for unpaired data. The chi-square test was used for categorical data. Spearman correlation analysis was used to assess the statistical relationships between the time intervals or MPI by TDE and the conventional method. A difference was considered significant at $P<0.05$.

\section{RESUlts}

Doppler time intervals: The basic clinical characteristics and Doppler time intervals for the healthy subjects and patients with previous MI are given in Table I. In patients with previous MI, ejection fraction was significantly decreased, IVCT was significantly prolonged, and ET was shortened in comparison with those in the healthy age-matched subjects. The IVRT was not significantly different between the two groups. The MPI measured either by TDE or the conventional method was significantly greater in patients with MI (Table II). 
Table I. Clinical Characteristics and Doppler Echocardiography Measurements in Healthy Subjects and Patients with MI

\begin{tabular}{lccc}
\hline \multicolumn{1}{c}{ Variable } & Healthy & MI & $\begin{array}{c}P \\
\text { value }\end{array}$ \\
\hline Age (y) & $61 \pm 7$ & $61 \pm 9$ & NS \\
Male/Female (no.) & $(11 / 4)$ & $(22 / 3)$ & NS \\
E to A ratio & $1.01 \pm 0.2$ & $0.94 \pm 0.3$ & $\mathrm{NS}$ \\
Deceleration time (ms) & $186 \pm 15$ & $189 \pm 10$ & $\mathrm{NS}$ \\
Ejection fraction (ms) & $68 \pm 4$ & $45 \pm 9$ & 0.0001 \\
Isovolumetric contraction time (ms) & $29 \pm 6$ & $50 \pm 17$ & 0.0001 \\
Ejection time (ms) & $316 \pm 10$ & $300 \pm 32$ & 0.045 \\
Isovolumetric relaxation time (ms) & $78 \pm 8$ & $70 \pm 12$ & $\mathrm{NS}$ \\
MPI (conventional) & $0.34 \pm 0.03$ & $0.40 \pm 0.1$ & 0.021 \\
MPI by TDE & $0.34 \pm 0.03$ & $0.48 \pm 0.09$ & 0.0001 \\
\hline
\end{tabular}

NS = Non-significant MPI = Myocardial performance index $;$ TDE $=$ Tissue

Doppler echocardiography; $\mathrm{ms}=$ Millisecond.

Table II. MPI Assessed by DTI at Different Sites of Mitral Annulus

\begin{tabular}{llllll}
\hline & MPI-Septum & MPI-Anterior & MPI-Lateral & MPI-Inferior & MPI-Mean \\
\hline Healthy subjects & $0.31 \pm 0.04$ & $0.34 \pm 0.04$ & $0.36 \pm 0.06$ & $0.35 \pm 0.05$ & $0.34 \pm 0.03$ \\
Patients with anterior MI $(n=14)$ & $0.54 \pm 0.14 \dagger \dagger$ & $0.54 \pm 0.16 \dagger \dagger$ & $0.45 \pm 0.15$ & $0.43 \pm 0.15$ & $0.49 \pm 0.11 \dagger \dagger$ \\
Patients with inferior MI $(n=11)$ & $0.46 \pm 0.10 \dagger \dagger$ & $0.35 \pm 0.07$ & $0.43 \pm 0.11$ & $0.59 \pm 0.10 \dagger \dagger$ & $0.46 \pm 0.07 \dagger \dagger$ \\
All patients with MI & $0.51 \pm 0.13 \dagger \dagger$ & $0.46 \pm 0.16 \dagger \dagger$ & $0.44 \pm 0.13 \dagger$ & $0.50 \pm 0.15 \dagger \dagger$ & $0.48 \pm 0.09 \dagger \dagger$ \\
\hline
\end{tabular}

MPI = Myocardial performance index; TDE = Tissue Doppler echocardiography; $\dagger \dagger P<0.01$ compared with healthy subjects, $\dagger P<0.05$ compared with healthy subjects

MPI in healthy subjects: There was no significant difference in the mean MPI by TDE values at four cardiac sites (Table II). There was a highly significant correlation between MPI values obtained by TDE and the conventional method ( $r=$ $0.81, P<0.0001$ ) (Figure 4). The IVRT, IVCT, and ET values obtained by TDE all correlated well with the values obtained by the conventional method $(r=0.80$, $P<0.0001 ; P<0.0001, r=0.91 ; P<0.0001, r=0.83, P<0.0001$; respectively) (Figures 5, 6, and 7).

MPI in patients with previous MI: There was a significant correlation between the MPI values obtained by TDE and the conventional method $(P<0.001, r=$ 0.62) (Figure 8). The IVRT, IVCT, and ET values obtained by TDE all correlated significantly with the values obtained by the conventional method $(r=0.49, P<$ $0.05 ; r=0.56, P<0.05 ; r=0.81, P<0.0001$; respectively) (Figures 9, 10, and $11)$.

In patients with anterior MI, the rise in MPI by TDE values was found to be more pronounced at the anterior and septal sites of the mitral annulus (Table II). 


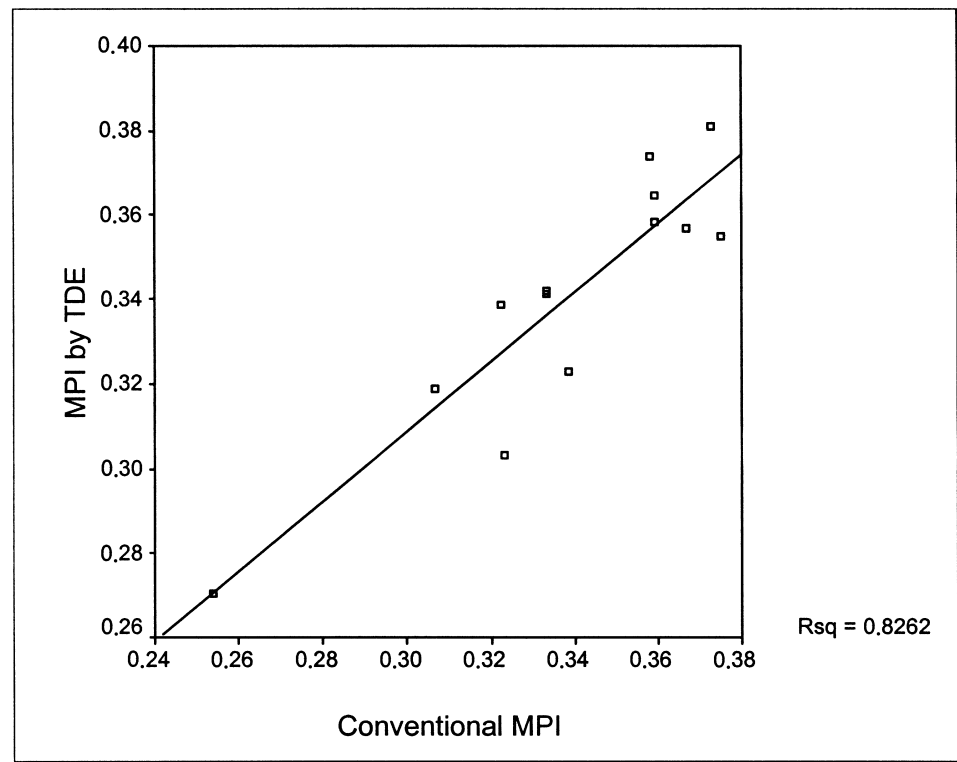

Figure 4. Correlation between MPI determined by TDE and conventional method in healthy subjects. MPI $=$ Myocardial performance index TDE $=$ Tissue Doppler echocardiography .

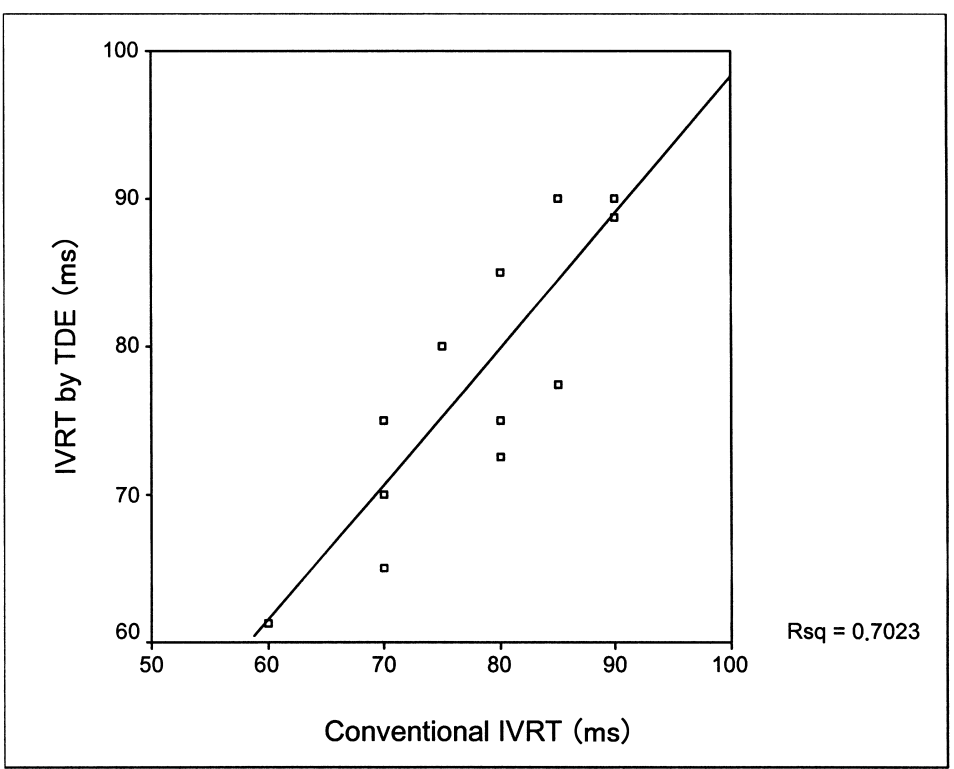

Figure 5. Correlation between IVRT determined by TDE and conventional method in healthy subjects. IVRT = Isovolumetric relaxation time; MPI = Myocardial performance index; TDE $=$ Tissue Doppler echocardiography. 


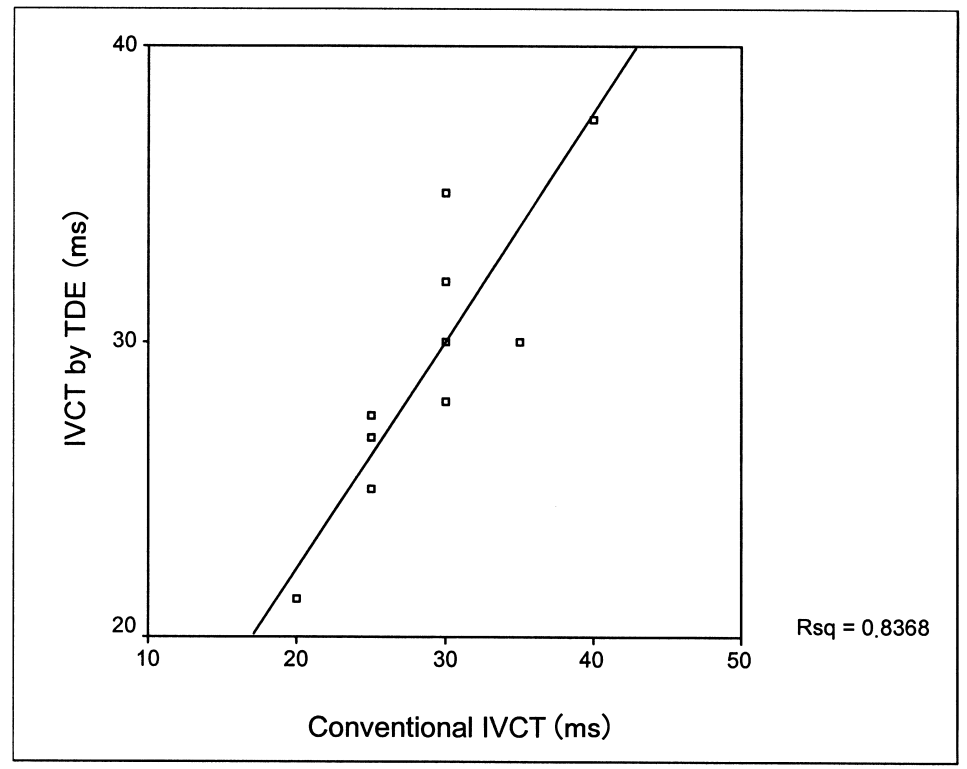

Figure 6. Correlation between IVCT determined by TDE and conventional method in healthy subjects. IVCT $=$ Isovolumic contraction time; MPI $=$ Myocardial performance index; TDE $=$ Tissue Doppler echocardiography.

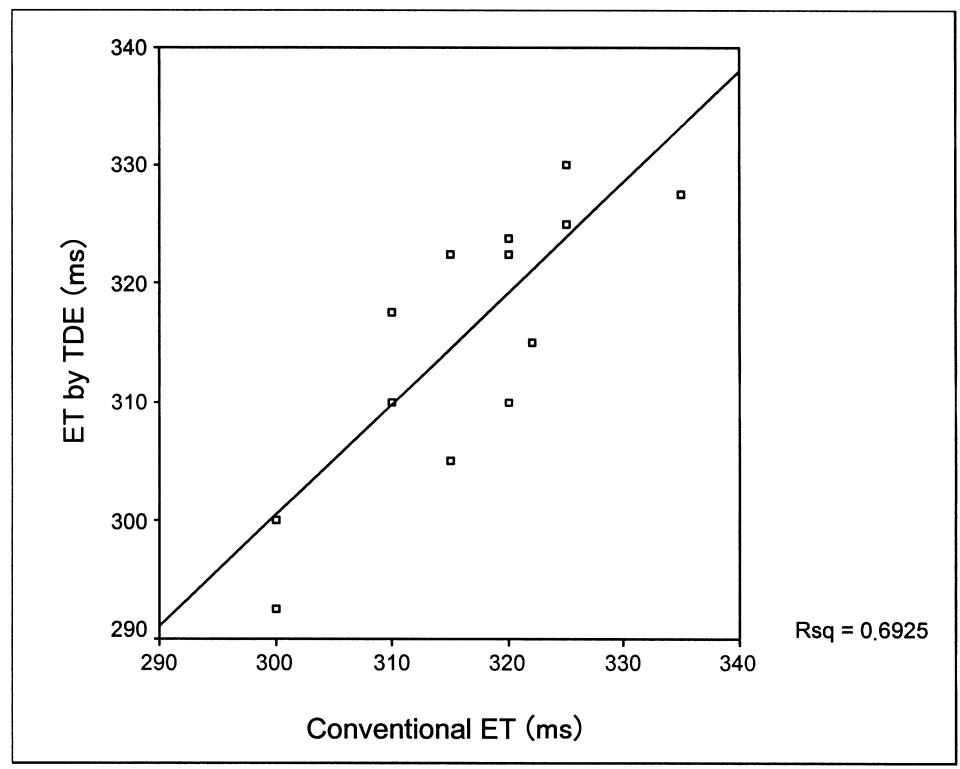

Figure 7. Correlation between ET determined by TDE and conventional method in healthy subjects. ET = Ejection time; TDE = Tissue Doppler echocardiography. 


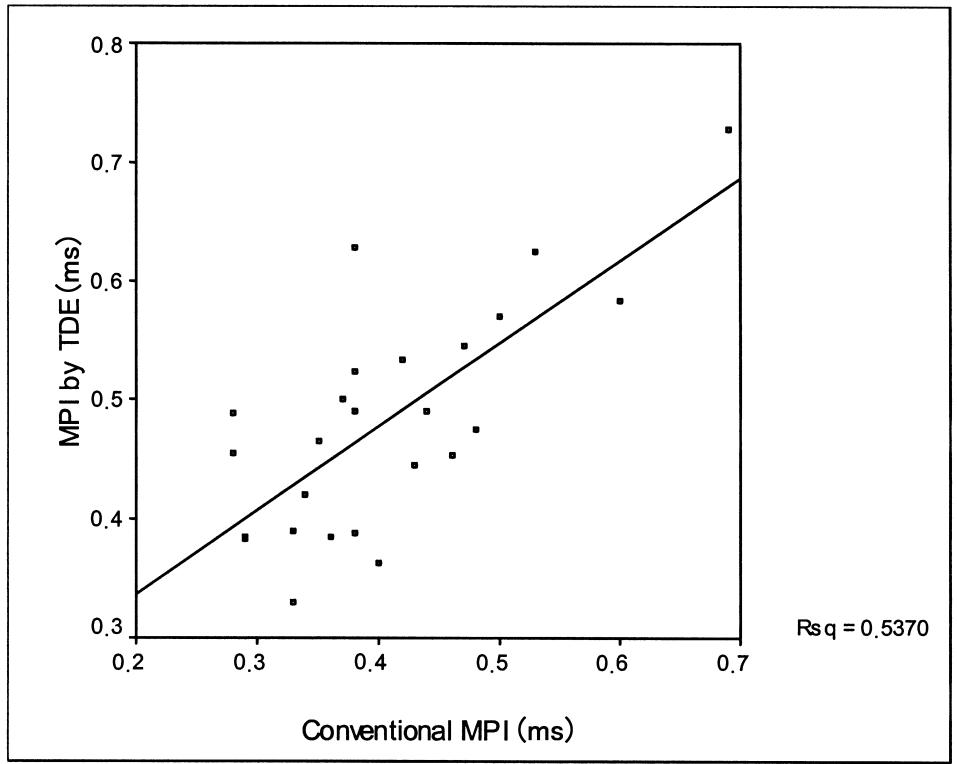

Figure 8. Correlation between MPI determined by TDE and conventional method in patients with MI MPI = Myocardial performance index; TDE = Tissue Doppler echocardiography; $\mathrm{MI}=$ Myocardial infarction.

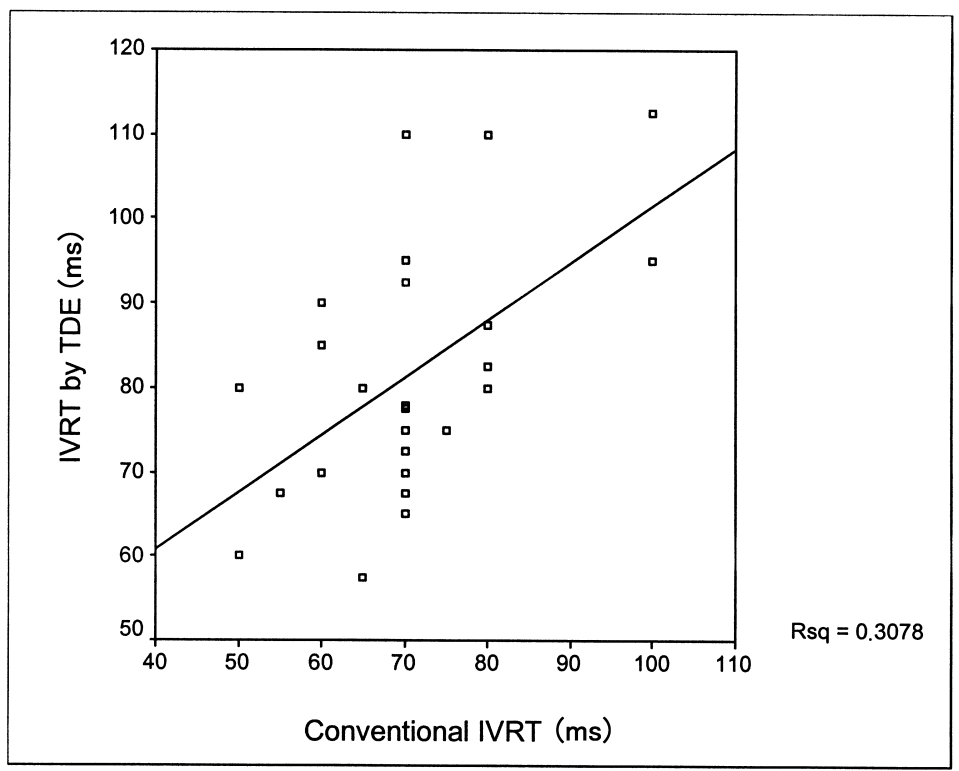

Figure 9. Correlation between IVRT determined by TDE and conventional method in patients with MI. IVRT = Isovolumetric relaxation time; MPI $=$ Myocardial performance index: TDE $=$ Tissue Doppler echocardiography; MI = Myocardial infarction. 


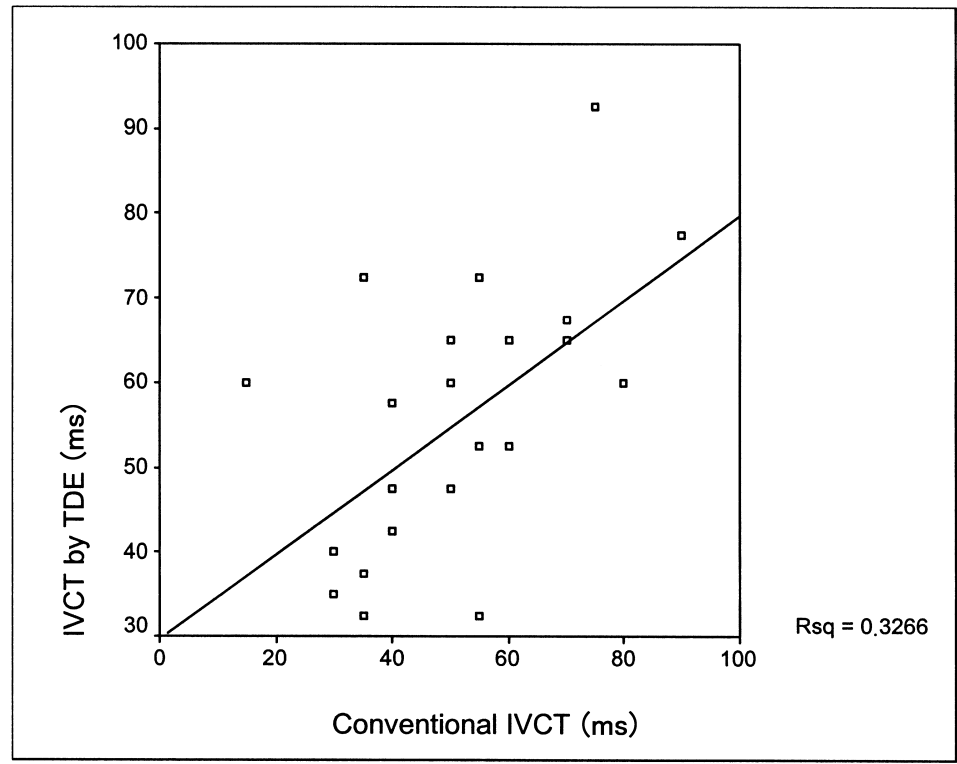

Figure 10. Correlation between IVCT determined by TDE and conventional method in patients with MI. IVCT = Isovolumetric contraction time; MPI = Myocardial performance index; MI = Myocardial infarction.

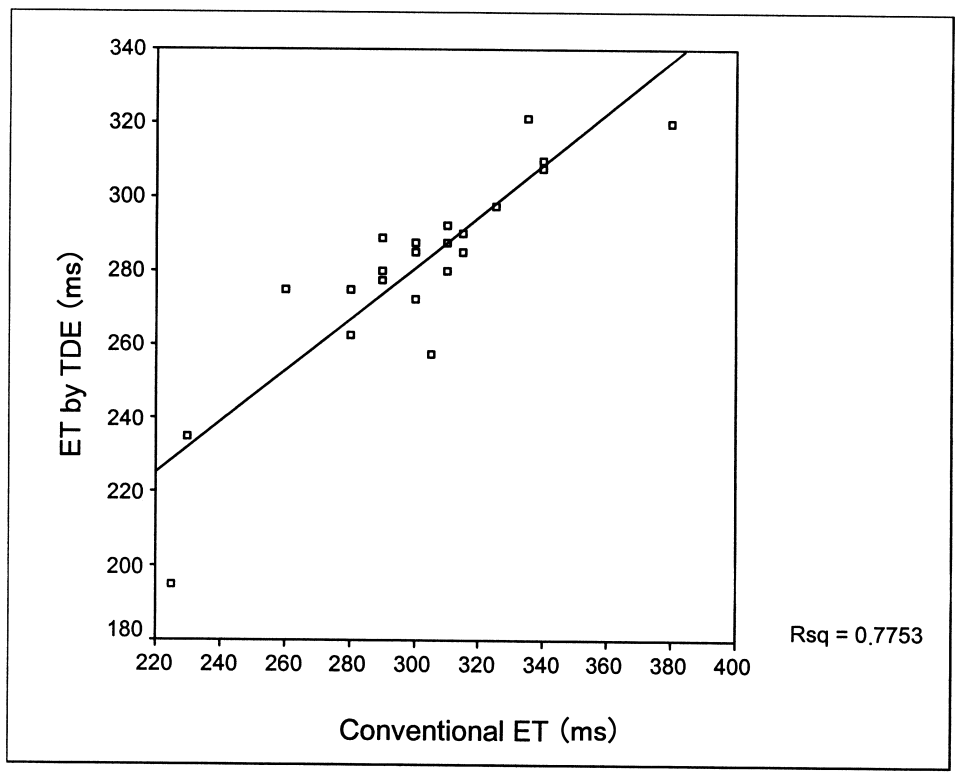

Figure 11. Correlation between ET determined by TDE and ejection fraction in patients with MI. ET = Ejection time; MPI = Myocardial performance index; MI = Myocardial infarction. 


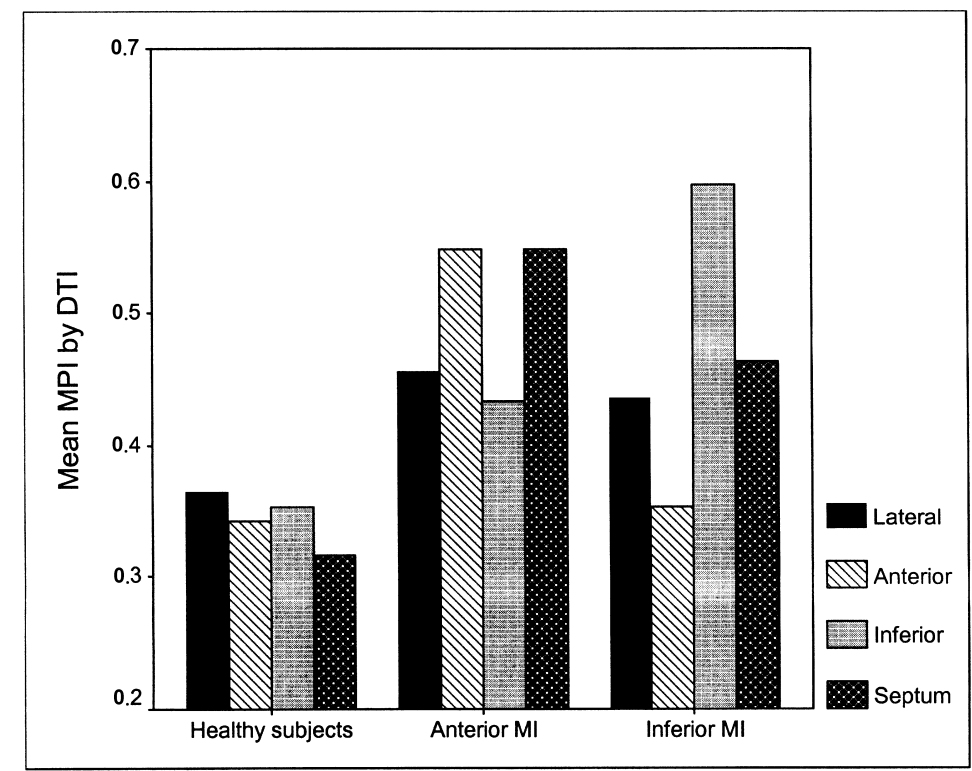

Figure 12. MPI determined by TDE at different sites of mitral annulus in healthy subjects and patients with anterior and inferior MI. MPI = Myocardial performance index; TDE $=$ Tissue Doppler echocardiography; $\mathrm{MI}=$ Myocardial infarction .

In patients with inferior MI, the rise in MPI by TDE values was found to be more pronounced at the inferior and septal sites of the mitral annulus (Table II) (Figure 12).

Reproducibility: Intra- and interobserver variability for Doppler derived parameters (IVRT, IVCT, ET) ranged from $2.4 \%$ to $5.8 \%$. Intraobserver variability for conventional MPI was $3.6 \pm 2.8 \%$ while interobserver variability was $3.6 \pm 2.1 \%$. For the TDE measurements (IVRT, IVCT, ET), intra- and interobserver variability ranged from $2.6 \%$ to $5.5 \%$. Intraobserver variability for MPI by TDE was 3.4 $\pm 2.6 \%$ and interobserver variability was $4.2 \pm 1.6 \%$.

\section{DISCUSSION}

In the present study, the Doppler intervals and MPI determined either by TDE or a conventional method were measured in age-matched healthy subjects, and the results were compared with those of patients with previous MI. To the best of our knowledge, this study was the first to use TDE in measuring MPI in patients with previous MI.

MPI values obtained using either method were significantly higher in the patients with previous MI (Table I). The prolongation of IVCT and the shortening 
of ET were the alterations in the time intervals that resulted in the increase in MPI values in patients with MI compared to the findings in the healthy age-matched subjects, which is consistent with the results of previous studies. ${ }^{89}$ )

In order to evaluate the applicability of MPI by TDE in assessing LV function, we used four sites in the mitral annulus to measure tissue Doppler time intervals. Left ventricular contraction involves both a reduction of the short axis diameter and a shortening along the longitudinal axis of the chamber. ${ }^{10,11)}$ The longitudinal systolic shortening of the left ventricle is reflected by the motion of the mitral annulus toward the cardiac apex in systole, whereas its recoil away from the apex is the result of diastole. As there is no appreciable motion of the apex in relation to the imaging transducer, the magnitude of the mitral annular motion reflects the extent of myocardial shortening along its longitudinal axis. ${ }^{11)}$ Recording the mitral annular motion has the advantage that it is devoid of trabeculea, myocardial dropouts, etc, and therefore is independent of echo quality. The major advantage of pulsed-wave mitral annular velocity measurements is that the ultrasound beam is parallel to the LV contraction. Moreover, it probably measures the transmural myocardial velocity, not only the epicardial and endocardial velocities. The introduction of pulsed-wave TDE at different mitral annular sites of the left ventricle opens up a new possibility. Thus, the pulsed-wave TDE parameters may be used as an additional method that could increase the accuracy of echocardiographic LV studies.

Although it continued to be statistically significant, the correlation between the two methods of measuring MPI, TDE and conventional, in patients with previous MI was not as high as it was in healthy individuals. This is due to the lesser degree of correlation between the IVRT and IVCT values (Figures 9 and 10). The presence of regional diastolic and systolic dysfunction might be the reason for less correlation in patients with previous MI. The goal of conventional MPI is to assess global LV function. If a single site is used for measurement, MPI by TDE is actually assessing LV function in that region. This may explain the higher MPI by TDE values in patients with previous MI who had LV wall motion abnormalities. In order to be able to assess global LV function, we used four sites in the mitral annulus for MPI by TDE measurements.

Conventional MPI has been reported to be independent of heart rate and blood pressure. However, the inability to measure the interval between the end and onset of mitral inflow and the ejection time simultaneously is a major limitation of conventional MPI. Due to this limitation, the results are probably less reliable in the presence of physiologic heart rate changes during examination. The TDE used in this study can simultaneously record systolic and diastolic mitral annular velocities. In contrast to the pulsed Doppler method, the time interval between the end and onset of diastolic annular velocities and the duration of the 
systolic wave can be measured in the same cardiac cycle. Thus, MPI by TDE may reduce inaccuracy due to heart rate fluctuation and has practical advantages over the conventional MPI. The other possible disadvantage of conventional MPI is the effect of loading conditions on conventional MPI. Tei, et al ${ }^{12)}$ found a high correlation between conventional MPI and peak dp/dt, suggesting a relationship between MPI and preload. Thus, even when contractility is constant, significant changes in preload may cause significant alterations in conventional MPI. Measurement of MPI by TDE may not be altered by preload changes. Further studies are needed to confirm this hypothesis.

Myocardial infarction is characterized by variable degrees of LV systolic and diastolic function, which have both been reported to have independent prognostic value. ${ }^{13,14)}$ A significant regional difference was found in the MPI at the LV sites related to the infarction site. Anterior MI patients had higher MPI values at the anterior site and septum than at other sites. The increased MPI by TDE values at the infarction site is an expression of myocardial damage after an MI. In inferior MI, the mean MPI values were not only increased at the inferior wall, but also at the interventricular septum (Table II). This may be the result of partial damage to the septum in the inferior MIs, as the posterior part of the septum is usually supplied by the right coronary artery. ${ }^{15)}$ The MPI at the noninfarction sites was also increased compared with healthy subjects (Table II). It is difficult to explain this phenomenon. We do not know whether the spirally oriented myocardial fiber, which causes the shortening of the left ventricle along its long axis, ${ }^{16)}$ might have some action beyond the infarction area.

Limitations: This study analyses the usefulness of MPI determined by pulsedwave TDE by using the myocardial velocity along the long axis. No consideration was taken of contraction of the left ventricle along its short axis caused by circumferential fibers. Another limitation is that coronary and LV angiography were not performed to assess and localize the LV wall asynergy. However, infarction sites were determined by both ECG and 2D echocardiography.

Conclusion: This study demonstrated that MPI can be measured by tissue Doppler and that it correlated well with conventional MPI. Moreover, MPI by TDE has the advantage of assessing both regional and global myocardial performance, and also has the ability to record systolic and diastolic velocity patterns simultaneously.

\section{REFERENCES}

1. Appleton C, Hatle L, Popp R. Relation of transmitral flow velocity patterns to left ventricular diastolic function: new insight from a combined hemodynamic and Doppler echocardiographic study. J Am Coll Cardiol 1988; 12: $426-40$. 
2. Galiuto L, Ignone G, DeMaria AN. Contraction and relaxation velocities of the normal left ventricle using pulsed-wave tissue Doppler echocardiography. Am J Cardiol 1998; 81: 609-14.

3. Yamazaki N, Mine Y, Sano A, et al. Analysis of ventricular wall motion using color-coded tissue Doppler imaging system. Jpn J Appl Physiol 1994; 33: 3141-6.

4. Tei C, Ling LH, Hodge DO, et al. New index combined systolic and diastolic myocardial performance: a simple and reproducible measure of cardiac function: a study in normals and dilated cardiomyopathy. J Cardiol 1995; 26: 357-66.

5. Tei C. New non-invasive index for combined systolic and diastolic function. J Cardiol 1995; 26: 396-404.

6. Tei C, Dujardin KS, Hodge DO, Kyle RA, Tajic AJ, Seward BJ. Doppler index combining systolic and diastolic myocardial performance: clinical value in cardiac amyloidosis. J Am Coll Cardiol 1996; 28: 658-64.

7. Schiller NB, Shah PM, Crawford M, et al. Recommendations for quantitation of the left ventricle by twodimensional echocardiography. J Am Soc Echocardiogr 1989; 2: 358-67.

8. Kurado T, Seward JB, Rumberger JA, Yanagi H, Tajik AJ. LV volume and mass: a comparative study of twodimensional echocardiography and ultrafast computed tomography. Echocardiography 1994; 11: 1-9.

9. Poulsen SH, Jensen SE, Tei C, Seward JB, Egstrup K. Value of Doppler index of performance in the early phase of acute myocardial infarction. J Am Soc Echocardiogr 2000; 13: 723-30.

10. Rushmer RF. Cardiovascular Dynamics. 4th ed. Philadelphia: WB Saunders; 1976: 93.

11. Lundback S. Cardiac pumping and the function of the ventricular septum. Acta Physiol Scand 1986; 550: 1101.

12. Tei C, Nishimura AR, Seward JB, Tajik AJ. Noninvasive Doppler-derived myocardial performance index: Correlation with simultaneous measurements of cardiac catheterization measurements. J Am Soc Echocardiogr 1997; 10: 169-78.

13. White HD, Norris RM, Brown AM, Brandt PW, Whitlock RM, Wild CJ. LV end - systolic volume as the major determinant of survival after recovery from MI. Circulation 1987; 76: 44-51.

14. Pfeffer AM, Braunwald E, Moye LA, et al. Effect of captopril on mortality and morbidity in patients with LV dysfunction after MI: results of the Survival and Ventricular Enlargement Trial. N Engl J Med 1992; 327: 66977.

15. Anatomy of the Heart. Schlant RC, Silverman ME, Robert WC. In Hurst JW, Schlant RC, editors. 7th ed. New York: McGraw-Hill Book Company; 1990: 26.

16. Rushmer RF. Cardiovascular Dynamics. 2nd ed. Philadelphia: WB Saunders; 1968: 34-6. 